\title{
Research on Optimal Excitation and Measurement Mode of Electrical Resistance Tomography
}

\author{
*LI Kun ${ }^{1, a}$, XU Lei, ${ }^{2, b}$, ZHANG Yu-lin ${ }^{3, c}$, PAN Zhi-hao ${ }^{4, d}$, AN Yu ${ }^{5, e}$ \\ (Shanxi Key Laboratory of Signal Capturing \& Processing, North University of China, Taiyuan, \\ 030051, China.) \\ alk_nuc@163.com, 'bl_nuc@163.com, 'yulin824@yeah.net, \\ dpanzh725@163.com, eanyuer321@163.com
}

Keywords: Electrical Resistance Tomography; excitation mode; measurement mode

\begin{abstract}
Excitation and measurement mode of Electrical Resistance Tomography (ERT) system would determine the sensitivity, information quantity and noise immunity of the test field. In this paper, a 16-electrode ERT system is cited to conduct a research on 8 excitation modes and 2 measurement modes by numerical analysis. The independent data volume, equipotential line distribution, as well as amplitude and sensitivity characteristics of measured voltage are separately compared. Considering the overall various factors, Mode7 and the reference measurement mode are considered to be the best choices.
\end{abstract}

\section{Introduction}

Electrical resistance tomography (ERT) technology is a kind of process tomography (PT) technologies, which is based on electrical sensitivity principle. In view of different medium with different conductivity, the medium distribution within test field could be speculated according to the object's conductivity distribution, thus fulfilling visualization measurement of test field [1]. Because ERT technology features high speed, low cost, no interference, no radiation and visualization, it has been widely used in multi-phase flow measurement in various fields, such as biomedicine, petroleum, chemical, food and agriculture industries[2-5].

For ERT system, excitation and measurement mode is classified into three patterns. The first is to measure the boundary voltage by injecting current into the test field. The second is, on the contrary, to measure the electrode current through inputting voltage signal. The last is to measure the induction current distribution that generated by an excitation coil. Considering that the second pattern is always seriously affected by contact impedance, while the third pattern has the weakness of low imaging precision, the first one is of course well popular among researchers presently[6]. Excitation mode refers to how the exciting current is injected into the test field, and common modes[7] include adjacent method, cross method and relative method, which are all based on the dual-electrode excitation method. Jiang et al. put forward a single electrode excitation method[8], in which electrode current is calculated by detecting the voltage of the standard resistance that connected to the electrode; Demidenko et al. proposed adaptive optimal excitation method[9], where a plurality of electrodes are stimulated simultaneously to obtain the best current distribution. Measurement mode refers to the voltage measurement method of boundary electrode, and general measurement modes involve adjacent measurement and reference measurement[10]. For the same system, different excitation and measurement modes directly impact on the sensitivity, the amount of measurement information and anti-noise performance of test field, even the final imagery quality.

For 8 excitation modes and 2 measurement modes based on the dual-electrode excitation method, the independent data volume, equipotential line distribution, as well as amplitude characteristic and sensitivity characteristic of measured voltage are compared respectively. All these indicators taken into consideration, the optimal excitation and measurement mode would be selected for the ERT system, which to an extent provides a reference for the design of ERT system. 


\section{Excitation and measurement modes of ERT system}

ERT system diagram is shown in Fig. 1. The main control unit takes control of excitation signal generation unit to produce a signal, which, through the electrode gated control unit, is injected into the test filed. Simultaneously, the data acquisition and processing unit collects voltage of electrodes via electrode gated control unit. And the imaging unit is designed to reconstruct the image from the measured data. The electrode gated control unit dominates which electrodes to be selected, in other words determining the excitation and measurement mode for ERT system.

Excitation mode defines the way of current injection and current distribution within test field. Taking 16-electrode ERT system for example, there are totally 8 excitation modes based on dual-electrode. One is the two excitation electrodes stand next to each other (such as 1-2 electrodes, noted as Mode1).The second mode is that there is another electrode between the two electrodes (such as 1-3 electrodes, Mode2), and so on. Obviously the seventh mode is that there are another 6 electrodes between the two electrodes (such as 1-8 electrodes, Mode7). The last mode is that the two electrodes are opposite (such as 1-9 electrodes, Mode8). Measurement mode is critical to features of measured voltage amplitude and anti-noise. As stated before, it mainly involves adjacent measurement mode and reference measurement mode. The adjacent measurement mode signifies measuring voltage between adjacent electrodes. The reference measurement mode means measuring voltage between the other electrode and the reference one which is selected from electrodes except the excitation electrode. Excitation and measurement modes of ERT system jointly decide the quality of reconstruction image.

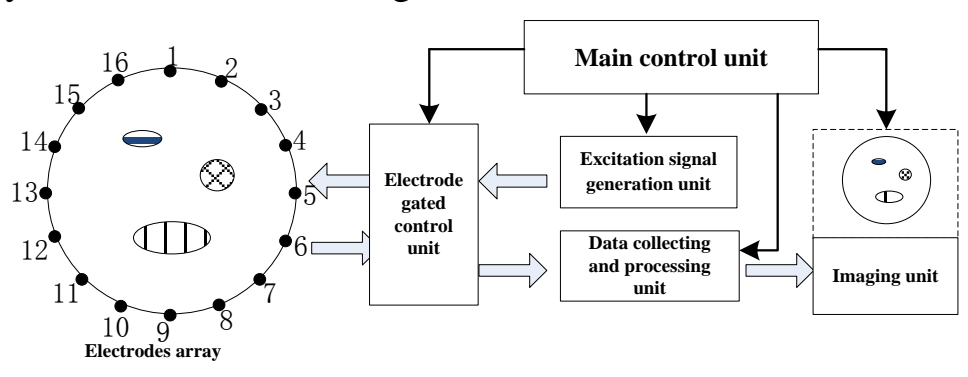

Fig. 1 Structure diagram of typical ERT system

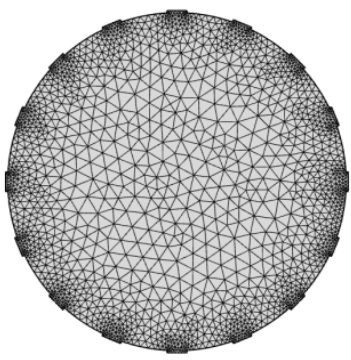

Fig. 2 Finite element mode

\section{Evaluation indexes of excitation and measurement modes}

In this paper, numerical simulation is adopted to carry out the research. First, COMSOL 5.1 software is employed to construct ERT test field model, where the radius of the pipe is R (79mm), and 16 electrodes, with the size of $0.15 \times \frac{2 \pi R}{16}$ [11], are located around the pipe, which form an ERT sensor array. Here we illustrated by the case of gas-liquid two-phase flow. The conductivity of liquid phase and gas phase are separately $1 \mathrm{~S} / \mathrm{m}$ and 0 , and the excitation current is set as $1 \mathrm{~mA}$, while the frequency is $50 \mathrm{kHz}$. When the ERT sensitivity field dissected with mesh, the finite element model could be obtained. As shown in Fig. 2, it contains 4512 triangle units and 292 triangular boundary elements.

\section{Independent data volume}

Many researchers had put forward the idea that the reciprocity of sensitivity field could halve the data volume of voltage measurement. For the adjacent excitation mode (Mode1), when in sensitive field with homogeneous medium, the independent data volume would be cut by half due to the electrode reciprocity. Namely when we exchange the excitation and measurement electrodes, the voltage value will remain the same; while in sensitive field with inhomogeneous medium, swapping the two electrodes will lead to change in voltage value. It implicates the nonreciprocity of electrodes in inhomogeneous medium and all the data is valid. Nonreciprocity also exists in Mode2 8. However, Mode8 has the property of structure symmetry, namely as to 1 and 9 electrodes, no matter which one is used as current input electrode or output electrode, the measured voltage value is 
exactly unchanged, which results in the size of independata volumedent data in half. The 16-electrode ERT system is cited to demonstrate independent data volume under different modes and the figures are listed in Table 1.

ERT inverse problem is an underdetermined nonlinear problem. Images that reconstructed from more independent data will enjoy higher resolution. From Table 1 we can see that, in inhomogeneous sensitivity field, Mode1 7 have more independent data, while the data volume of Mode8 suffers serious decline, so Mode8 do not perform as well as the others. When employing adjacent measurement pattern, mode1 is the best choice. When applying the reference measurement mode, there is little difference among Mode1 7 for they share the same amount of independent data.

Table 1 Volume of independent data in different modes

\begin{tabular}{|c|c|c|c|c|}
\hline \multirow{2}{*}{$\begin{array}{c}\text { Excitation } \\
\text { mode }\end{array}$} & \multicolumn{2}{|c|}{ Adjacent measurement mode } & \multicolumn{2}{c|}{ Reference measurement mode } \\
\cline { 2 - 5 } & $\begin{array}{c}\text { Homogeneous } \\
\text { field }\end{array}$ & $\begin{array}{c}\text { Inhomogeneous } \\
\text { field }\end{array}$ & $\begin{array}{c}\text { Homogeneous } \\
\text { field }\end{array}$ & $\begin{array}{c}\text { Inhomogeneous } \\
\text { field }\end{array}$ \\
\hline Mode1 & 104 & 208 & 208 & 208 \\
\hline Mode2 & 192 & 192 & 208 & 208 \\
\hline Mode3 & 192 & 192 & 208 & 208 \\
\hline Mode4 & 192 & 192 & 208 & 208 \\
\hline Mode5 & 192 & 192 & 208 & 208 \\
\hline Mode6 & 192 & 192 & 208 & 208 \\
\hline Mode7 & 192 & 192 & 208 & 104 \\
\hline Mode8 & 92 & 96 & 104 & \\
\hline
\end{tabular}

\section{Equipotential lines distribution}

Excitation mode specifies the way of current injection and current distribution within test field. The equipotential line corresponding to each mode is shown in Fig. 3. Excitation electrode interval increasingly grows from Mode1 to 8 and the equipotential lines distribute more and more uniformly. Mode1 is more sensitive to medium changes on the edge, but less sensitive at the center. With the electrode interval increasing, the equipotential lines distribute more and more uniformly, which further improves the property of soft field, and enhances its sensitivity to center change. The equipotential lines in Mode8 enjoy the most uniform distribution, which means the Mode8 is the best choice, followed by Mode7.
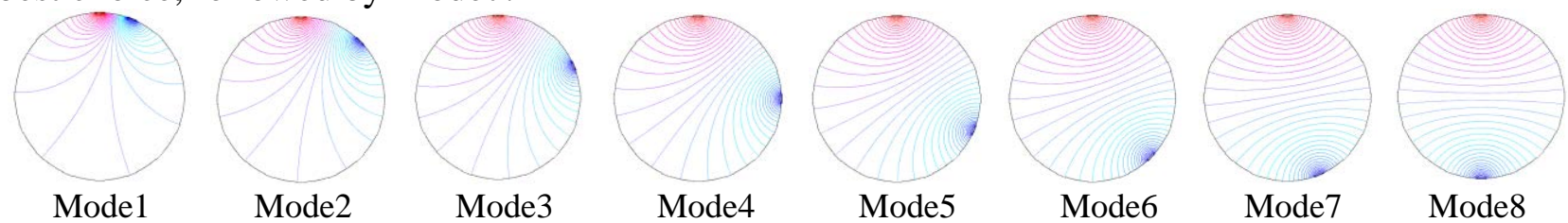

Fig. 3 Distribution of equipotential lines

\section{Amplitude of measurement voltage}

Since the source and amplitude of noise do not change along with the observed signal, the signal noise ratio (SNR) will be larger if boundary voltage has a larger value, which implies the bigger minimum and average measured voltage will result in better performance. The dynamic voltage range is theoretically defined as $D R=20 \lg \frac{\max \left(U_{\text {mear }}\right)}{\min \left(U_{\text {mear }}\right)}$. But in actual measurement, the system enjoys higher measurement accuracy with narrower dynamic voltage range. That's to say the voltage range should be as small as possible. In test field with homogeneous medium, boundary voltage is measured under different mode respectively. And its average value, maximum value, minimum value as well as the dynamic range are respectively presented in Table 2. 
Table 2 Measurement data under various modes

\begin{tabular}{|l|c|c|c|c|c|c|c|c|}
\hline & \multicolumn{2}{|c|}{ Maximum value (V) } & \multicolumn{2}{|c|}{ Minimum value (V) } & \multicolumn{2}{|c|}{ Mean value (V) } & \multicolumn{2}{c|}{$\begin{array}{c}\text { Dynamic range DR } \\
\text { (dB) }\end{array}$} \\
\cline { 2 - 9 } & adjacent & $\begin{array}{c}\text { reference } \\
\text { resources }\end{array}$ & adjacent & $\begin{array}{c}\text { reference } \\
\text { resources }\end{array}$ & adjacent & $\begin{array}{c}\text { reference } \\
\text { resources }\end{array}$ & adjacent & $\begin{array}{c}\text { reference } \\
\text { resources }\end{array}$ \\
\hline Mode1 & 0.00955 & 0.11042 & 0.00122 & 0.06788 & 0.00327 & 0.08915 & 17.88712 & 4.22561 \\
\hline Mode2 & 0.01385 & 0.14431 & 0.00251 & 0.07760 & 0.00556 & 0.11096 & 14.83552 & 5.38903 \\
\hline Mode3 & 0.04328 & 0.16398 & 0.00380 & 0.08177 & 0.01046 & 0.12288 & 21.13007 & 6.04351 \\
\hline Mode4 & 0.03353 & 0.17688 & 0.00525 & 0.08429 & 0.01330 & 0.13058 & 16.10549 & 6.43836 \\
\hline Mode5 & 0.02931 & 0.18541 & 0.00671 & 0.08607 & 0.01515 & 0.13574 & 12.80587 & 6.66522 \\
\hline Mode6 & 0.02678 & 0.19069 & 0.00851 & 0.08752 & 0.01633 & 0.13910 & 9.95762 & 6.76471 \\
\hline Mode7 & 0.02497 & 0.19323 & 0.01032 & 0.08880 & 0.01699 & 0.14101 & 7.67408 & 6.75353 \\
\hline Mode8 & 0.02351 & 0.19323 & 0.01285 & 0.09002 & 0.01720 & 0.14163 & 5.24662 & 6.63461 \\
\hline
\end{tabular}

From table 2 and Fig. 4, we can conclude that under adjacent measurement mode, with electrode interval improving (i.e. Mode1 Mode8), the minimum and average voltage gradually increase and the dynamic range suffers a decrease in general. As regard to the signal to noise ratio and dynamic range, Mode8 is proved to be the optimal excitation mode, followed by Mode7. In case of reference measurement mode, with the increase in electrode interval, voltage value grows gradually, and the voltage dynamic range expands too, however, the voltage values under different excitation modes are more or less the same. Mode 8 is supposed to be the optimum based on comprehensive consideration of SNR and dynamic range. Compared with adjacent measurement, the value of measured voltage under reference mode increases by about 10 times, and dynamic ranges under the two modes almost remain the same, which obviously proves that the reference measurement has a better performance.
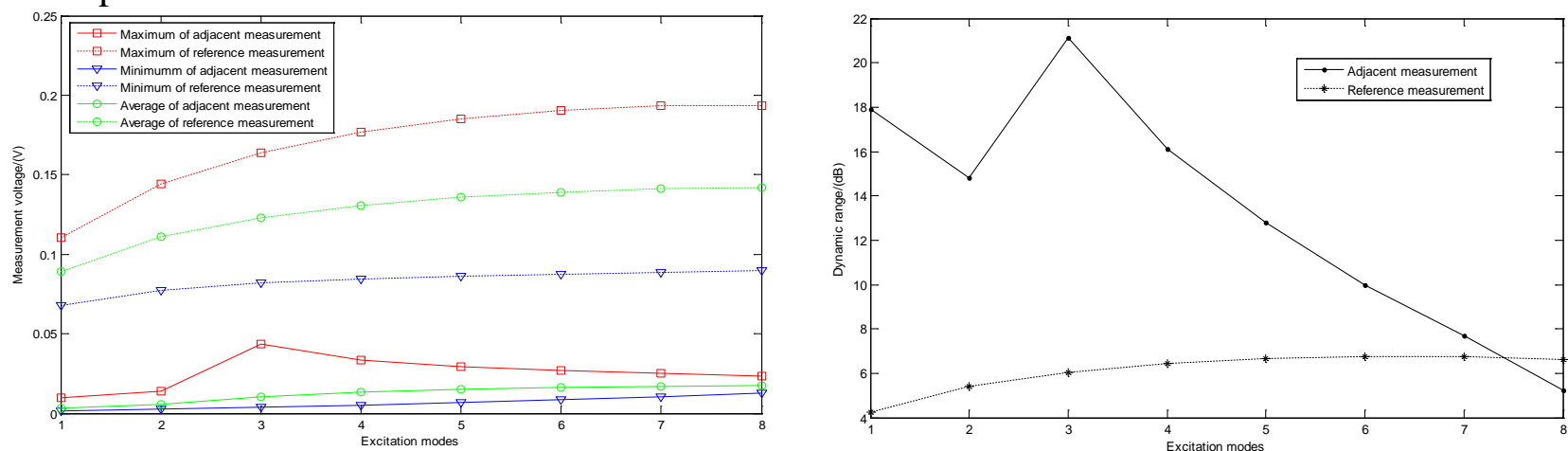

(a) Boundary measurement voltage

(b) The dynamic range of boundary measurement voltage Fig. 4 Amplitude characteristic and dynamic range of the measuring electrode

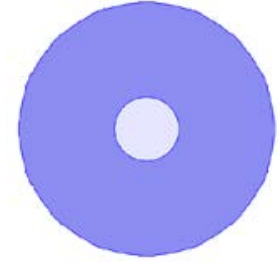

Style1

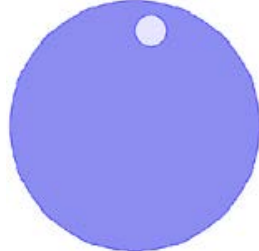

Style2

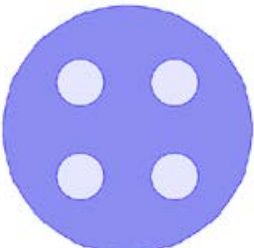

Style3

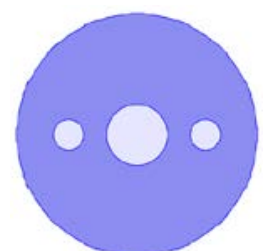

Style4

Fig. 5 Bubble flow modes

\section{Sensitivity of measurement voltage}

When the conductivity distribution in test field experiences a change, boundary voltage will alter as well. In this paper, the voltage sensitivity is quantified with standard deviation. For the same measurement system, higher voltage sensitivity means better detection of the system, and the corresponding mode is supposed to be better choice. The standard deviation of boundary voltage is 
defined as $S D=\sqrt{\frac{1}{N} \sum_{i=1}^{N}\left(u_{i 1}-u_{i 0}\right)^{2}}$, in which $u_{i 0}$ and $u_{i 1}$ separately represent the boundary voltage of homogeneous field and two-phase flow field.

As shown in Fig. 5, four bubbly flow models were researched in this paper. In Style1 model, there was a bubble at the center with the radius of $20 \mathrm{~mm}$. In Style2 model, a bubble with the radius of $10 \mathrm{~mm}$ located near one electrode, and four bubbles with the radius of $15 \mathrm{~mm}$ distributed evenly in Style3 model. While in Style4 model, there was a bubble with the radius of $20 \mathrm{~mm}$ at the center and two bubbles with the radius of $10 \mathrm{~mm}$ on its both sides. The standard deviation of the boundary voltage were calculated for each mode under various modes, the results were shown in Fig. 5.

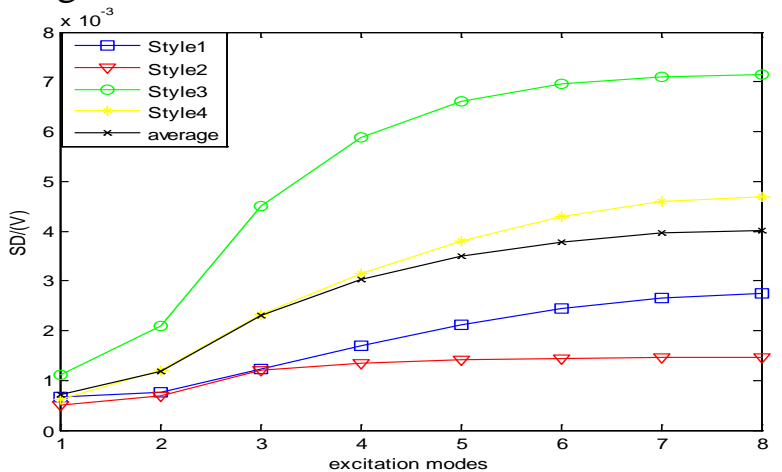

(a) Adjacent measurement mode

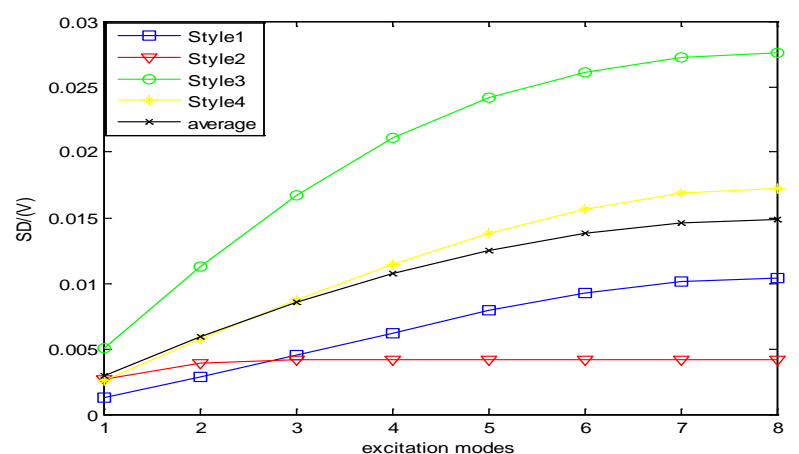

(b) Reference measurement mode

Fig. 6 Sensitivity of measured voltages in various modes

Fig. 6(a) and (b) separately showed the results of standard deviation and its average value of the mentioned four test fields under adjacent measurement and reference measurement modes. As the electrode interval increasing, voltage sensitivity under each mode increased gradually. Mode8 had the best performance and Mode 7 came the second, whose sensitivity almost remained the same as Mode8. Compared with adjacent measurement mode, the sensitivity of reference measurement mode increased around 5 times, which indicated the reference measurement mode was better than the adjacent measurement.

\section{Conclusion}

The excitation and measurement mode was connected with the quality of ERT imaging. In this paper, a 16-electrode ERT system was cited to carry out a research on 8 excitation modes and 2 measurement modes by numerical analysis. The independent data volume, equipotential line distribution, as well as amplitude and sensitivity characteristics of measured voltage were separately compared. Considering from the volume of independent data, Mode 8 had the worst performance, while Mode1 7 performed better and did not show much difference in data volume. What's more, the data volume of Mode1 7 were basically the same under adjacent and reference measurement modes. From equipotential line distribution, excitation Mode8 was the best choice, followed by Mode 7. In respect to the amplitude and sensitivity of measurement voltage, Mode8 was the optimal excitation and Mode7 took the second place, in addition, reference measurement mode performed better than the adjacent measurement. Based on all the above indicators, it was clearly concluded that the optimal excitation mode of ERT system was Mode7 (namely the two excitation electrodes are 6 electrodes apart), the optimal measurement mode was reference measurement mode.

\section{Acknowledgements}

This work was financially supported by the Natural Science Foundation of China (61227003), the Shanxi Natural Science Foundation (2012021011-2) . 


\section{References}

[1] Yasmin Abdul Wahaba, Ruzairi Abdul Rahimb, etc. Non-invasive process tomography in chemical mixtures-A review[J]. Sensors and Actuators B, 2015(210):602-617

[2] Wang Huaxiang. Electrical tomography [M]. Beijing: Science Press, 2013

[3] Baoliang Wang, Yuanyuan Hu.etc. A Novel Electrical Resistance Tomography System Based on C4D Technique[C]. IEEE TRANSACTIONS ON INSTRUMENTATION AND MEASUREMENT 2013,5, (62):1017-1024

[4] DONG Feng, XU Cong.etc. Design of Parallel Electrical Resistance Tomography System for Measuring Multiphase Flow[J].Chinese Journal of Chemical Engineering 2012,20, (2): 368-379

[5] Fan Wenru, Hao Kuihong, Xu Yanbin. Research on excitation strategy of open electrical impedance tomography[J]. Chinese Journal of Scientific Instrument,2014,6 (35): 1269-1275

[6] Xu Guizhi. Electrical impedance tomography in biomedical engineering[M]. Beijing: China Machine Industry Press, 2010

[7] Li Lipin, Dang Ruirong, Huang Yanqun. Review on tomography technologies in multiphase flow.[J]PROGRESS IN GEOPHYSICS, 2012,2 (27): 0651-0659

[8] Jiang Changzhen, Sun Yongjin, Xu Lingan. A New mode in Electrical Resistance Tomography[J]. Chinese Journal of Scientific Instrument, 2003,5 (24):517-524

[9] Eugene Demidenko, Hartov Alex, al. On Optimal Current Patterns for et Electrical Impedance tomography[J]. IEEE Transactions on Biomedical Engineering 2005,52, (2): 238-248

[10] He Wei, Luo Ciyong, Xu Zheng. Electrical Impedance tomography[M]. Beijing: China Machine Industry Press, 2009

[11] Wang Huaxiang, Wang Jing. Optimal Design of ERT/ECT Dual-Modality Sensing Electrode Array[J]. Journal of Tianjin University, 2008,8 (41): 911-918

[12] Luo Ciyong, He Wei, Dong Xiuzhen. Driven Patterns with Fixed Excitation in Electrical Impedance Tomography[J]. China Journal of Biomedical Engineering, 2009, 28 (6): 949-953 